\title{
Mulheres Primigestas: um olhar sobre o desempenho ocupacional e o novo papel social de mãe
}

\author{
Primiparous Women: a look at occupational performance and the new social role of mother \\ Mujeres primíparas: una mirada al desempeño ocupacional y el nuevo rol social de la madre
}

Priscila Barros Lourenço ${ }^{1 *}$, Renilce Machado dos Santos Araújo ${ }^{1}$, Jorgeane Pedrosa Pantoja ${ }^{1}$, Lívia Sue Saito de Oliveira Toda ${ }^{1}$, Elson Ferreira Costa ${ }^{1}$, Luísa Sousa Monteiro Oliveira².

\begin{abstract}
RESUMO
Objetivo: Analisar o desempenho ocupacional e o novo papel social de mãe de mulheres primigestas. Métodos: Trata-se de um estudo transversal, descritivo, exploratório, quanti-qualitativo, com 10 mulheres primigestas, na faixa etáriaentre17 e 37 anos (com média de idade $=26,7$ ), atendidas em um Centro de Saúde Escola, no período de julho e agosto de 2021. Resultados: No desempenho ocupacional as categorias que apresentaram diferenças nas médias foram: vestuário, sistema urinário e transferência. Quanto desempenhar o papel social de mãe, a maioria apresentou dúvidas relacionadas à futura rotina de cuidados com o bebê. Conclusão: Pode-se observar que o desempenho ocupacional da mulher primigesta tem comprometimento em pelo menos uma área de AVD e que o papel social de mãe ainda é visto como complexo, uma vez que o pré-natal ainda está focado mais no processo de gestação em si, assim pretende-se reforçar que a mulher primigesta receba orientações para melhor desempenhar suas tarefas ocupacionais e de seu bebê ainda durante o pré-natal.
\end{abstract}

Palavras-chave: Gestantes, Análise e desempenho de tarefas, Terapia ocupacional.

\section{ABSTRACT}

Objective: To analyze the occupational performance and the new social role of mothers of primiparous women. Methods: This is a cross-sectional, descriptive, exploratory, quantitative-qualitative study with 10 primiparous women, aged between 17 and 37 years (mean age $=26.7$ ), attended at a School Health Center, from July to August 2021. Results: In occupational performance, the categories that showed differences in the averages were: clothing, urinary system and transfer. As for playing the social role of mother, most had doubts related to the future routine of baby care. Conclusion: It can be observed that the occupational performance of the primiparous woman is compromised in at least one area of ADL and that the social role of a mother is still seen as complex, since prenatal care is still more focused on the process of pregnancy itself, so it is intended to reinforce that the primiparous woman receives guidance to better perform her occupational tasks and that of her baby even during prenatal care.

Key words: Pregnant women, Task performance and analysis, Occupational therapy.

\section{RESUMEN}

Objetivo: Analizar el desempeño ocupacional y el nuevo rol social de las madres de mujeres primíparas. Métodos: Se trata de un estudio transversal, descriptivo, exploratorio, cuantitativo-cualitativo con 10 primíparas, con edad entre 17 y 37 años (edad media $=26,7$ ), atendidas en un Centro de Salud Escolar, entre Julio y agosto de 2021. Resultados: En el desempeño ocupacional, las categorías que presentaron diferencias en los promedios fueron: vestuario, aparato urinario y traslado. En cuanto a desempeñar el papel social de madre, la mayoría tenía dudas relacionadas con la futura rutina del cuidado del bebé. Conclusión: Se puede observar que el desempeño ocupacional de la mujer primípara se encuentra comprometido en al menos un área de las AVD y que el rol social de una madre aún es visto como complejo, ya que el prenatal aún está más centrado en el proceso del propio embarazo, por lo que se pretende reforzar que la mujer primípara reciba orientación para desempeñar mejor sus tareas laborales y la de su bebé incluso durante el prenatal.

Palabras clave: Mujeres embarazadas, Análisis y desempeño de tareas, Terapia ocupacional.

1 Universidade do Estado do Pará (UEPA), Belém - PA. *E-mail: tolourencopb@gmail.com

2 Universidade Federal do Pará (UFPA), Belém - PA.

SUBMETIDO EM: 1/2022

ACEITO EM: 2/2022

PUBLICADO EM: 2/2022 


\section{INTRODUÇÃO}

A história de vida da mulher irá influenciar diretamente na construção do seu ser mãe, podendo se tornar numa experiência fácil ou difícil, lembrando que cada gestação acontece de maneira diferente e com significados variados, gerando assim um ser único (ZANETTINI A, et al., 2019).

Como o processo da maternidade inicia muito antes da concepção, teóricos compreendem a gestação como a preparação para a maternidade. Logo, a gravidez é um momento extremamente importante na reestruturação na vida da mulher e nos papéis que esta exerce onde é preciso manejar as mudanças em diversas esferas: biológicas, somáticas, psicológicas e sociais (PICCININI CA, et al., 2008; MARQUES ACM; LF SOUZA, 2019).

Na esfera biológica a gravidez se inicia antes mesmo da gestação, começando a ser contada desde o primeiro dia da última menstruação. Passando pelos períodos de fecundação, embrionário e fetal. Onde ocorrerá o encontro de óvulo e espermatozoide, formando a célula Ovo ou Zigoto, que se multiplicará e se movimentará até chegar ao útero, onde se originará o Embrião, este se desenvolverá e dará origem ao Feto respectivamente (MOORE KL, et al., 2016; SADLER TW, 2016).

Didaticamente a gestação pode ser dividida em 3 etapas: primeiro trimestre (de 0 a 13 semanas), onde há o preparo da mulher para a gravidez, seu corpo sofre modificações necessárias e alguns sentimentos e sensações também são comuns acontecerem, como a dualidade de negação-aceitação e a satisfaçãoincômodo naturais do início da gravidez, por exemplo. No segundo trimestre (de 14 a 26 semanas) a barriga começa a crescer, os primeiros movimentos surgem, iniciando os laços af etivos mãe-bebê e as sensações de incômodo dão lugar às de conforto. Já no terceiro e último trimestre (de 27 a 40, 41,42 semanas) o útero passa a ser pequeno para comportar o bebê, causando sensações naturais do fim da gravidez como o desconforto, a mulher também pode ficar mais ansiosa ou emotiva devido o p arto estar próximo (MINISTÉRIO DA SAÚDE, 2018).

A mulher primigesta segundo Fernandes $F$, et al. (2019) é aquela que tem sua primeira gestação. $E$ independentemente de sua idade gestacional, essa nova experiência envolve sensações sentimentais, de atitude e que repercutirá em transformações de ordem biológica e psicoemocional, onde também a mulher terá que se adaptar a uma nova rotina (NUNES G, et al., 2018).

Segundo O Ministério da Saúde (2018) as puérperas primigestas principalmente podem de imediato se sentir inseguranças com a chegada de um bebê, o que é normal, pois a rotina da casa mudará completamente e uma rede de apoio é essencial, se possível, para ajudar a controlar esse sentimento. A mulher agora terá um novo papel, o papel social de mãe (FERREIRA-SANTOS E, 1997).

O homem é um ser ocupacional. E considerando que durante o período gestacional e/ou pós-gestacional a mulher passa por mudanças que necessita de adaptação para desempenhar suas Atividades de Vida Diária (AVD's) entre outras tarefas, o Terapeuta Ocupacional (TO) vem contribuir positivamente para que ela possa ter autonomia e independência para realizar suas atividades ocupacionais normalmente (NASCIMENTO C, et al., 2017).

O TO durante as suas intervenções foca diretamente no desempenho ocupacional que segundo Pedretti L e Early M. (2005) pode ser classificado como a habilidade de um indivíduo enquanto ser ocupacional em desempenhar suas tarefas de forma significativa. Assim este estudo teve como objetivo analisar 0 desempenho ocupacional e o novo papel social de mãe de mulheres primigestas.

\section{MÉTODOS}

Trata-se de um estudo com delineamento transversal, de caráter descritivo, do tipo exploratório e com abordagem quanti-qualitativa dos dados. A pesquisa foi realizada com 10 mulheres primigestas, na faixa etária entre 17 e 37 anos (com média de idade = 26,7), atendidas em um Centro de Saúde Escola (CSE) de uma universidade de um município do Pará, no período de julho e agosto de 2021. O critério de seleção deste Centro de Saúde Escola e das participantes foi por amostragem não probalística do tipo intencional ou julgamento. 
Foram considerados como critérios de Inclusão: gestantes e puérperas primigestas cadastradas no prénatal do CSE e mulheres que sofreram aborto anteriormente, sendo a gestação atual considerada como "primeira". Já os critérios de exclusão foram: mulheres cujo filho primogênito tenha ido a óbito após seis meses de vida.

Uma ficha de entrevista semiestruturada foi utilizada para avaliar o papel social de mãe, dividida em dados pessoais (nome, idade, trimestre gestacional, escolaridade, estado civil, etc.) e o seguinte questionamento: Quanto desempenhar o papel social de mãe, quais suas maiores dúvidas? E para analisar o desempenho ocupacional foi aplicada a Escala Índice de Barthel (IB), qual avaliou as seguintes AVD's: alimentação, banho, atividades rotineiras, vestir-se, controle insfecteriano (intestino), controle insfecteriano (bexiga), uso do toilet, transferência (da cama para a cadeira e vice-versa), mobilidade (em superfícies planas) e escadas.

$\mathrm{Na}$ Escala IB cada item recebe o valor mínimo de 0 ponto e máximo de 15 pontos, distribuídos da seguinte forma: valor mínimo de 0 ponto e máximo de 5 pontos nos seguintes itens (banho e atividades rotineiras), valor mínimo de 0 ponto e máximo de 10 pontos nos seguintes itens (alimentação, vestir-se, intestino, sistema urinário, uso do toilet e escadas) e valor mínimo de 0 ponto e máximo de 15 nos seguintes itens (transferência e mobilidade). Quanto menor a pontuação, maior será o grau de dependência e vice-versa. A pontuação total é a soma de todos os itens avaliados do IB, a depender do grau de capacidade que o indivíduo considera desempenhar a tarefa, obtendo-se assim o grau de dependência, conforme segue: <20 (total); 20-35 (grave); 40-55 (moderada); $\geq 60$ (leve) e 100 (independente) (RUZAFA JC e MORENO JD, 1997).

A coleta de dados ocorreu em quatro etapas: na primeira as participantes foram esclarecidas sobre a pesquisa, na segunda as que aceitaram participar da pesquisa assinaram voluntariamente 0 Termo de Consentimento Livre e Esclarecido (TCLE), na terceira foi aplicada a ficha de entrevista e a escala IB e na quarta etapa as participantes receberam uma cartilha impressa elaborada pelas autoras e orientações sobre 0 assunto abordado.

Quanto ao questionário os dados pessoais foram analisados por análise estatística descritiva. Já para o questionamento as entrevistadas foram enumeradas de 1 a 10 e suas respostas foram transcritas na íntegra e analisadas de acordo com análise do conteúdo de Bardin (2016) que é dividida em três etapas: pré-análise, exploração do material e tratamento dos resultados, inferência e interpretação e a análise do desempenho ocupacional adquirida através da Escala de Barthel foi tabulada em planilhas do pacote estatístico IBM Statistica Packagefor the Social Science (SPSS) na versão 20), verificados dados de média e desvio padrão a partir da associação com a variável trimestre gestacional, para posterior análise e comparações dos resultados.

O estudo levou em consideração os aspectos éticos da resolução 466/2012 do Conselho Nacional de Saúde (CNS) que regulariza as pesquisas que envolvam seres humanos e foi aprovado pelo Comitê de Ética em Pesquisa (CEP) da Universidade do Estado do Pará (UEPA), CAAE № 43369821.4.0000.5174.

\section{RESULTADOS E DISCUSSÃO}

\section{Dados pessoais}

Como resultados em relação aos dados pessoais coletados das 10 participantes (100\%), quanto: à faixa etária1 (10\%) tinham menos de 20 anos, $3(30 \%)$ entre 21 e 25 anos, $4(40 \%)$ entre $26-30$ anos e $2(20 \%)$ mais de 30 anos. Pesquisas realizadas em 2017 e 2019 também obtiveram como resultado o maior número de gestantes primigestas na faixa etária superior a 20 anos (NUNES GS, et al., 2018; COSTA S, et al., 2019).

Em relação ao trimestre gestacional $1(10 \%)$ se encontravam no primeiro, $4(40 \%)$ no segundo e $5(50 \%)$ no terceiro. De acordo com o Ministério da Saúde (2018) ao aumentar o número de semana de gestação o período de intervalo entre as consultas vai diminuindo, frequentemente serão encontrados maiores números de gestantes a partir de seis meses para realizar consulta pré-natal, pesquisa com o mesmo público também registraram resultados parecidos (COSTA S, et al., 2019).

Tratando-se de escolaridade: $4(40 \%)$ possuíam ensino médio e $6(60 \%)$ ensino superior e à questão de estado civil: $5(50 \%)$ são solteiras,2(20\%) vivem em uma união estável e $3(30 \%)$ são casadas. Mulheres tornando-se mães com escolaridade mínima de ensino médio completo e em sua maioria solteiras foram 
registrados em estudos de Nunes GS, et al. (2018) e de mães na sua maioria com ensino médio ou superior completo e casadas em estudos de Costa S, et al. (2019). De acordo com Bruzamarello D, et al. (2019) a ascensão profissional feminina e quebras dos ditos padrõ es culturais, além da idade tardia das primigestas são fatores que contribuem para esses resultados.

\section{Desempenho ocupacional}

O Desempenho Ocupacional envolve o cliente, seu contexto e ocupação, sendo analisado quanto competências e limitações ou potenciais problemas do cliente segundo Gomes D, et. al. (2021) e pode ser avaliado durante o pré-natal em uma unidade básica de saúde por um profissional TO. O resultado da análise do desempenho ocupacional está apresentado na Tabela 1.

Tabela 1 - Análise do Desempenho Ocupacional de Mulheres Primigestas atendidas em um Centro de Saúde Escola $(n=10)$.

\begin{tabular}{lccc}
\hline Trimestre Gestacional & Primeiro & Segundo & Terceiro \\
\hline Categoria & & & \\
\hline Alimentação M(dp) & $10(0)$ & $10(0)$ & $10(0)$ \\
Banho M(dp) & $5(0)$ & $5(0)$ & $5(0)$ \\
Atividades Rotineiras M(dp) & $5(0)$ & $5(0)$ & $5(0)$ \\
Vestuário M(dp) & $5(0)$ & $6,3(2,5)$ & $5(0)$ \\
Intestino M(dp) & $10(0)$ & $10(0)$ & $10(0)$ \\
Sistema Urinário M(dp) & $10(0)$ & $10(0)$ & $9(2,2)$ \\
Uso do Toillet & $10(0)$ & $10(0)$ & $10(0)$ \\
Transferência & $15(0)$ & $13,8(2,5)$ & $15(0)$ \\
Mobilidade & $15(0)$ & $15(0)$ & $15(0)$ \\
Escadas & $10(0)$ & $10(0)$ & $10(0)$ \\
\hline
\end{tabular}

Fonte: Lourenço PB, et. al., 2022.

Quanto às categorias as que apresentaram diferenças nas médias foram: vestuário, sistema urinário e transferência.

Associadas com a variável trimestre gestacional o desempenho no vestuário foi mais af etado em mulheres que estão no segundo trimestre gestacional. O Ministério da Saúde (2018) afirma que durante a gestação ocorrem mudanças físicas como o crescimento da barriga, tornando o vestuário, principalmente de MMII uma das AVD's bastante comprometida. (MARQUES KR, et al., 2002)

A cadeira adequada para gestante se colocar na posição sentada, elevando as pernas, fletir o joelho e apoiar por cima do outro membro, serve como recurso auxiliar para facilitar no Vestuário do MMII. (NASCIMENTO CRF, et al., 2017).

Já o desempenho no sistema urinário foi mais af etado em mulheres que estão no terceiro trimestre gestacional. O Ministério da Saúde (2018) afirma que é comum no início e ao fim do processo da gravidez dar mais vontade de ir ao banheiro urinar e isso se deve ao fato de que com o aumento do útero para abrigar o feto, a bexiga sofre maior pressão, por isso algumas mulheres no último mês de gestação podem apresentar alguma perda de urina. (SOUZA APP, et al., 2016)

A adaptações ambientais apresentadas por Teixeira IC (2008), banheiro localizado próximo à cama da gestante, uso de urinol e em alguns casos a necessidade do uso de fralda. Além de recomendações focadas no equilíbrio funcional para que AVD's, lazer, entre outros sejam realizadas com melhor desempenho, são uma das possíveis orientações dadas pelo TO (MARQUES KR, et al., 2017).

E o desempenho de transferência foi mais afetado em mulheres que estão no segundo trimestre gestacional. Para Teixeira IC (2008) conforme cresce a barriga a barriga da gestante, a postura passa a ser comprometida, passando a aumentar as dores na coluna, segundo o Ministério da Saúde (2018), af etando o desempenho da transferência conforme afirma Marques KR, et al. (2002) como a do deitar/levantar da cama que foi uma das respostas de uma participante. 
Segue algumas das orientações de TO para adaptação nas transferências da gestante para deitar: devese sentar na beira da cama, sobre os cotovelos ir apoiando o tronco, girar de costas, colocar as pernas na cama. Para levantar da cama (recomenda-se preparar as articulações, lubrificando-as com movimentos circulares) e seguir as seguintes etapas: virar de lado, sobre os cotovelos ir apoiando o tronco (sem flexionalo) e ir colocando as pernas para fora da cama. (TEIXEIRA IC, 2008)

Considerando o grau de dependência das 10 participantes (100\%) a partir da classificação da escala IB: $2(20 \%)$ alegaram independência e $8(80 \%)$ leve dependência. Como pode-se observar existem fatores associados à gestação que justificam esse resultado de dependência ocupacional em AVD's durante o período gestacional. (MARQUES KR, et al., 2002; TEIXEIRA IC, 2008; NASCIMENTO CRF, et al., 2017).

\section{Papel social de mãe}

Desempenhar o papel social de mãe, entre outras tarefas está em acompanhar a saúde da criança. Fatores biológicoseambientais influenciam diretamente no seu crescimento (variável quantitativa) e desenvolvimento (variável qualitativa). Ministério da Saúde (2020) nos traz que devido à grande plasticidade nos primeiros anos de vida, a vigilância de o desenvolvimento tornar-se essencial para estimulação e intervenção precoce. Sendo uma das demandas voltadas para o profissional TO dentro da Atenção Primária à Saúde (APS).

Ao serem questionadas quanto suas principais dúvidas para desempenhar o papel social de mãe, a maioria relatou dúvidas relacionadas à futura rotina de cuidados com o bebê em si (banho, umbigo, amamentação, limpeza da cavidade oral) e poucas relataram fazer pesquisas sobre o assunto de cuidados com o bebê, conforme suas falas transcritas apresentadas a seguir:

\section{Rotina de cuidados com o Bebê}

Os cuidados prestados ao bebê no domicilio geram dúvidas nas mães, sobretudo, nas mulheres primigestas por estarem vivendo a primeira experiência nos cuidados com recém-nascido, que apresenta dependência total dos seus cuidados. As mães frequentes relatam dúvidas no cuidado com o coto umbilical, dificuldade na amamentação e higiene, como vemos nos recortes a seguir:

"Tenho dúvidas quanto ao banho, limpeza da cavidade oral e cuidados com o coto umbilical do bebê" (Entrevistada 1).

"Considerando-se cuidados, tenho todas dúvidas (risos)" (E2).

"Tenho dúvidas principalmente quanto ao banho" (E3).

"Em relação à rotina do bebê, tenho muitas dúvidas" (E6).

"Aleitamento materno é o que mais me preocupa" (E7).

"Cuidados com o umbigo" (E8).

"Com a rotina de um bebê em si, mas estou dando tempo ao tempo, esperando o futuro" (E10).

A rotina na casa realmente muda, o novo integrante da família precisa de muitos cuidados, junto com sua mamãe, que precisará de dicas e apoio, o banho e o umbigo, por exemplo, ainda são vistos como cuidados difíceis de executar (TOMELERI KR e MARCON SS, 2009).

Orientações como: banho humanizado usando banheira, fralda, água morna etc., limpeza da cavidade oral com gaze e água filtrada ou fervida, limpeza do coto umbilical apenas com algodão ou gaze e álcool $70^{\circ}$ etc. devem ser dadas a mulher ainda durante o pré-natal, para que ela fique mais tranquila ao desempenhar 0 papel de mãe em relação à rotina do bebê (ORGANIZAÇÃO PAN-AMERICANA DA SAÚDE (OPAS), 2018; MINIST'RIO DA SAÚDE, 2017; 2018; 2020). Buscou-se of ertar na cartilha elaborada pelas autoras estas e outras orientações para as participantes.

A gravidez mesmo planejada traz muitas transformações para a mulher desde a descoberta até receber o bebê no colo para exercer o papel de mãe, o processo de nove meses vem para preparar a mulher para a 
maternidade, onde o terapeuta ocupacional pode estar contribuindo em todos os processos e no binômio mãe-bebê (MARQUES KR, et al., 2002; SOUSA TA, 2015; MINISTÉRIO DA SAÚDE, 2018).

\section{Pesquisa sobre $O$ assunto de cuidados com o bebê}

Por se tratar da primeira gravidez as mães vivem em um contexto de dúvidas, do quais muitas são sanadas no período do pré-natal e/ou permanece principalmente quando se deparam com os cuidado e rotina do bebê, com o acesso a informações as mães se dedicam a pesquisar nas mais diferentes fontes, como são expressadas nas falas a seguir:

"Eu tenho dúvidas, mas geralmente procuro estar me informando sobre como cuidar de um bebê" (E4).

"Como eu sou babá procuro sempre pesquisar sobre o assunto de rotina do bebê" (E5).

"Sou técnica de enfermagem e cuidadora de idosos, então sempre me atualizo sobre o assunto de cuidados com o bebê" (E9).

Exercer o papel social de mãe é muito mais que saber cuidar de uma criança, envolve vínculo, acompanhamento do crescimento e desenvolvimento entre outras tarefas (BARRETO APV e OLIVEIRA ZM, 2010; SOUSA TA, 2015; MINISTÉRIO DA SAÚDE, 2018).

As buscas por informações são bastante comuns e importantes, no entanto não se deve descartar a importância do acompanhamento pré-natal para tirar dúvidas com pessoas que realmente entendem do assunto (BARRETO APV e OLIVEIRA ZM, 2010; MINISTÉRIO DA SAÚDE, 2018).

Percebe-se assim que a gestação e maternidade são singulares e complexas, onde a mulher passa por intensas transformações biopsicossocial e emocional, por isso é importante uma rede de apoio: da unidade de saúde, companheiro, familiar e amigos, se possível. (MARQUES KR, et al., 2002; BARRETTO APV e OLIVEIRA ZM, 2010; ZANATTA E, et al., 2017; MINISTÉRIO DA SAÚDE, 20218).

O apoio da unidade de Saúde está diretamente ligado à Atenção Primária à Saúde - APS, sendo esta a porta de entrada do Sistema Único de Saúde (SUS) visando promoção da saúde, prevenção de agravos, proteção entre outros, tendo que ser ao máximo resolutiva (MINISTÉRIO DA SAÚDE, 2017).

A APS também é a responsável pelo primeiro acolhimento das mulheres que planejam ser mãe e das que engravidam sem planejamento, cujo processo perpassa pela avaliação pré-concepcional, pré-natal e pósparto, planejamento familiar e na continuidade do atendimento com o acompanhamento da saúde da mãe e do bebê visando o atendimento humanizado e integral da gestante (MINISTÉRIO DA SAÚDE, 2012; 2018).

Na equipe da APS o TO especialista em Saúde da Família é apto a atuar no acolhimento, diagnóstico, planejamento, traçar plano terapêutico, atendimento, reavaliação até a alta do sujeito, podendo também exercer cargo de Gestor entre outros de acordo com o Conselho Federal de Fisioterapia e Terapia Ocupacional (COFFITO) (2011) sendo também um dos profissionais hábil a trabalhar com o processo gestacional na saúde da mulher e da criança.

Segundo Mendes EV (2015) gestantes, puérperas e seus recém-nascidos são considerados como umas das condições crônicas e estas na perspectiva da APS necessitam de respostas e ações permanentes, eficaz e integral, envolvendo todo o sistema, os próprios profissionais e tornando estas mulheres também protagonistas do cuidado, objetivando cada vez mais melhoria, qualidade e resolutividade ainda no acompanhamento pré-natal destas mulheres.

\section{CONCLUSÃO}

Pode-se observar que o desempenho ocupacional da mulher primigesta tem comprometimento em pelo menos uma área de Atividade de Vida Diária e que o papel social de mãe ainda é visto como sendo complexo, uma vez que o pré-natal ainda está focado mais no processo de gestação em si, assim pretende-se reforçar 
que a mulher primigesta receba orientações para melhor desempenhar suas tarefas ocupacionais e de seu bebê ainda durante o pré-natal. O estudo buscou contribuir com as mulheres primigestas a partir da entrega de uma cartilha elaborada pelos autores com orientações sobre o assunto abordado, mostrando uma das importâncias do profissional TO na rotina de consultas do pré-natal na preparação da mulher primigesta para a chegada do seu bebê.

\section{REFERÊNCIAS}

1. BARDIN L. Análise de Conteúdo. São Paulo: Edições 70, 2016.

2. BARRETO APV, OLIVEIRA ZM. O ser mãe: expectativas de primigestas. Rev.Saúde.Com, 2010;6(1):9-23.

3. BRUZAMARELLO D, et al. Ascenção profissional feminina, gestação tardia e conjugalidade. Psicologia em Estudo, $2019 ; 24(4): 1-15$.

4. CONSELHO FEDERAL DE FISIOTERAPIA E TERAPIA OCUPACIONAL (COFFITO). Disciplina a Especialidade Profissional Terapia Ocupacional em Saúde da Família e dá outras providências. Resolução coffito no 407 de 18 de agosto de 2011.2011. Disponível em: https://www.coffito.gov.br/nsite/?p=3170. Acessado em:15 Fev. 2022.

5. COSTA S, et al. A prática do aleitamento materno na percepção de mulheres primigestas. Vivencias, 2019; 15(29): 289-310.

6. FERNANDES F, et al. A idade da primeira gestação no Brasil: dados da pesquisa nacional de saúde. J. Hum. Growth Dev., 2019;29(3):304-312.

7. FERREIRA-SANTOS E. Psicoterapia breve:abordagem sistematizada de situações de crise. $2^{\circledR}$ ed. São Paulo: Ágora, 1997.

8. GOMES D, et al. Enquadramento da Prática da Terapia Ocupacional: Domínio e Processo. 4ª ed. Leiria - Portugal: Politécnico de Leiria, 2021;1-73 p.

9. MARQUES ACM, SOUZA LF. Gestação e seus fatores emocionais. Trabalho de Conclusão de Curso - Centro Universitário de Anápolis. UniEvangélica, Anápolis, 2019;41p.

10. MARQUES KR, et al. A importância da terapia ocupacional no pré-parto, parto e puerpério.Multitemas, 2016;0(26).

11. MENDES EV. A construção social da atenção primáriaà saúde. Brasília: Conselho Nacional de Secretários de Saúde, 2015.

12. MINISTÉRIO DA SAÚDE. Aprova a Política Nacional de Atenção Básica, estabelecendo a revisão de diretrizes para a organização da Atenção Básica, no âmbito do Sistema Único de Saúde (SUS). Portaria Ministério nº 2.436, de 21 de Setembro de 2017. 2017.2 Disponível https://bvsms.saude.gov.br/bvs/saudelegis/gm/2017/prt2436 2209 2017.html. Acessado em:15 de Fev, de 2022.

13. MINISTÉRIO DA SAÚDE. Atenção Humanizada ao Recém-ñascido de Baixo Peso - Método Canguru. 2017. Disponível em: https://bvsms.saude.gov.br/bvs/publicacoes/atencao_humanizada_recem_nascido_canguru.pdf. Acessado em: 11 de Nov. de 2020.

14. MINISTÉRIO DA SAÚDE. Caderneta de saúde da criança (menino). 2020. Disponível em: https://portaldeboaspraticas.iff.fiocruz.br/wp-content/uploads/2020/09/caderneta_crianca_menino_2ed.pdf. Acessado em: 12 de Nov. 2020.

15. MINISTÉRIO DA SAÚDE. Caderneta da Gestante. 2018. Disponível em: https://portaldeboaspraticas.iff.fiocruz.br/wpcontent/uploads/2019/02/Caderneta-da-Gestante-2018.pdf. Acessado em: 13 de Nov. de 2020

16. MINISTÉRIO DA SAÚDE. Cadernos de Atenção Básica. Atenção ao Pré-Natal de Baixo Risco. 2012. Disponível em:

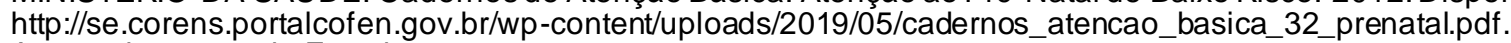
Acessado em: 15 de Fev. de 2022.

17. MOORE KL, et al. Embriologia clínica. 9ae ed. Rio de Janeiro:Elsevier, 2016.

18. ASCIMENTO CRF, et al. Ações de terapia ocupacional com gestantes na rotina diária. Rev. Interinst. Bras. Ter. Ocup., 2017;1(5): 556-573.

19. NUNES GS, et al. Sentimentos vivenciados por primigestas. Rev enferm UFPE, 2018; 12(4): 916-922.

20. ORGANIZAÇÃO PAN-AMERICANA DA SAÚDE (OPAS). Centro Latino-Americano de Perinatologia, Saúde da Mulher e Reprodutiva. Prevenção de infecções relacionadas à assistência à saúdeem neonatologia. 2018. Disponível em: https://portaldeboaspraticas.iff.fiocruz.br/wp-content/uploads/2018/04/clap1613-03.pdf. Acessado em: $18 \mathrm{de} \mathrm{Nov.}$ de 2020.

21. PEDRETTI L, EARLY M. Terapia Ocupacional: capacidade prática para as disfunções físicas. São Paulo: Roca, 2005.

22. PICCININI CA, et al. Gestação e a Constituição da maternidade. Psicologia em estudo, 2008;13 (1): 63-72.

23. RUZAFA JC, MORENO JD. Valoración de la discapacidad física: el indice de Barthel. Revista Española de Salud Pública, 1997; 71(2): 127-137.

24. SADLER TW. Langman embriologia médica. 13를. Rio de Janeiro: Guanabara Koogan, 2016.

25. SOUSA TA. Perspectivas de Atuação do Terapeuta Ocupacional na Linha de Cuidado Atenção a Saúde do Recémnascido. Trabalho de Conclusão de Curso- Centro de Ciências da Saúde, Universidade Federal da Paraíba, João Pessoa, 2015;49 p.

26. SOUZA APP, et al. Prevalência de incontinência urinária durante a gestação. Revista Baiana de Saúde Pública, 2016; 40(1): 216-228.

27. TEIXEIRA IC. 2008. In: Guia de Orientações Básicas de Terapia Ocupacional para Gestantes. SlideShare. Disponível em:https://pt.slideshare.net/isabelctf/guia-de-orientaes-bsicas-de-terapia-ocupacional-para-gestantes.Acessado em: 11 de nov. de 2020.

28. TOMELERI KR, MARCON SS. La madre adolescente cuidando al niño em la primer semana de vida. Revista Brasileira de Enfermagem, 2009;62 (3): 355-361.

29. ZANATTA E, et al. A experiência da maternidade pela primeira vez: as mudanças vivenciadas no tornar-se mãe. Pesquisas e Práticas Psicossociais, 2017; 12(3):1-16.

30. ZANETTINE A, et al. As vivências da maternidade e a concepção da interação mãe-bebê:interfaces entre as mães primíparas adultas e adolescentes. Revista Fundamental Care Online, 2019;11 (3): 655-663. 